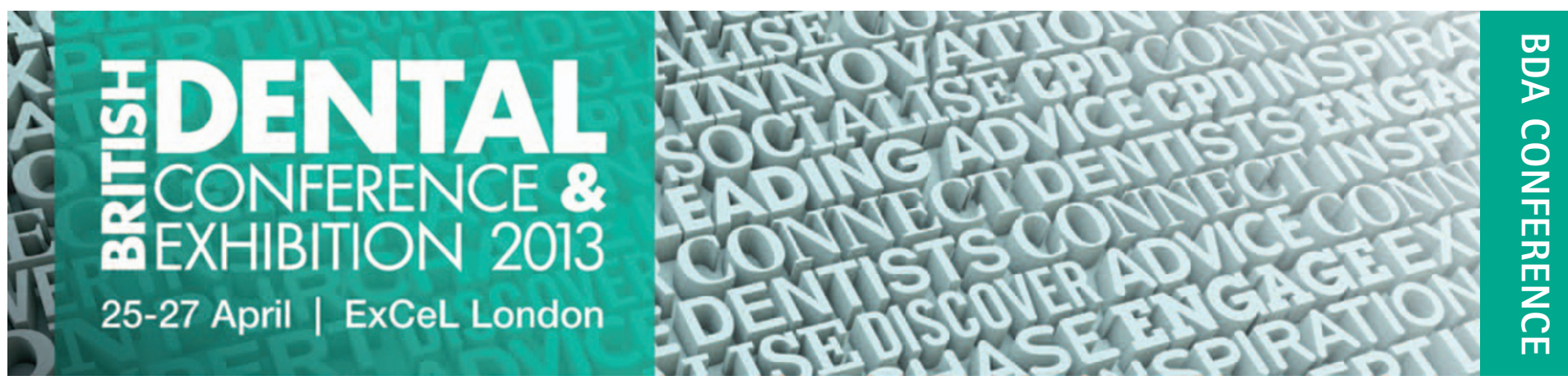

\title{
Short-term cosmetic orthodontics for general dental practitioners
}

\section{A. Maini ${ }^{1}$}

The demand for cosmetic dental treatments in the UK has grown dramatically in the last decade possibly due to increased public awareness of new techniques coupled with greater media attention for celebrities following smile enhancement treatments. In view of this, the aim of this article and the associated presentation at the 2013 British Dental Conference and Exhibition is to provide an overview of short-term cosmetic orthodontics for general dental practitioners. The presentation will cover the differences between short-term cosmetic orthodontics and comprehensive orthodontic treatment, and explain how using short-term orthodontics can be an effective tool to deliver minimally invasive cosmetic dentistry.

The demand for cosmetic dental treatments in the UK has grown dramatically in the last decade due to increasing public awareness of techniques available to enhance smiles and the media drawing attention to celebrities, such as Cheryl Cole who has had cosmetic smile enhancement treatments. An attractive smile has been shown to increase a person's confidence, improve their career prospects and ability to find a partner.

In recent years there has been a significant increase in general dentists using orthodontic appliances to assist them in treating patients who are unhappy with their smiles due to malaligned anterior teeth in relatively short timeframes of weeks or months.

Typically, these patients have only had two options in the past:

1. Traditional comprehensive orthodontic treatment

\footnotetext{
Vice President \&t Education Chair, British Academy of Cosmetic Dentistry and Clinical Director, Aqua Dental Spa, 25 Manchester Square, London, W1U 3PY

Correspondence to: Anoop Maini

Email:mainianoop@aol.com
}

DOI: $10.1038 /$ sj.bdj.2013.52

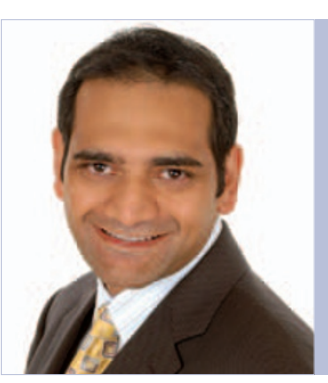

Annop Maini qualified from King's College in 1992 and has worked in private practice in London's West End since 1993. He has a special interest in advanced cosmetic and implant dentistry, having studied extensively in the USA and the UK. He currently serves on the Board of Directors of the British Academy of Cosmetic Dentistry.

Dr Maini was one of the first UK dentists to become a Six Month Smiles ${ }^{\circledR}$ provider and has been actively helping other dentists around the world learn and become more proficient with the system.

2. Restorative correction of malalignment using ceramic restorations.

\section{PROVIDING A CHOICE}

Most adult patients do not pursue comprehensive orthodontics, even though it is the gold standard, since they are not prepared to wear fixed appliances for years. They are only seeking correction to their crooked smiles and are not driven by correction of any posterior malocclusions.

In most cases, preparing teeth for ceramic restorations leads to aggressive removal of tooth structure which has a negative, long-term impact on the biomechanics of the tooth and the risks of pulpal damage. This is an option that many patients avoid due to the high costs and long-term irreversible damage to their teeth.

Therefore many of these adult patients would have had no realistic treatment options available to them. However, short-term orthodontics is an option for many of these patients by using aesthetic appliances over short time frames where the focus of movements are only on those teeth that show in the smile rather make corrections to the posterior occlusion. From experience, most adult patients will tolerate orthodontics provided the treatment is less than nine months.

Combining simple tooth alignment techniques with tooth whitening and incisal composite edge bonding techniques or non-preparation veneers allows general dentists to radically improve the symmetry and aesthetics of a smile in a 


\section{BDA CONFERENCE}

very minimally invasive and affordable way. This approach is a reasonable alternative option for patients who would not accept traditional orthodontics or a restorative ceramic solution.

\section{TYPES OF APPLIANCES}

There are several appliances which are simple to use and training is available for general dentists to become proficient with these techniques.

1. Removal appliances:

- Inman Aligner ${ }^{\circledR}$ - this is a NiTi coil activated appliance with clear anterior bars that compress the incisor teeth into alignment with a light continuous force. Some cases can be completed in as little as eight weeks.

- Clear aligners - these are a series of removable trays that fit over the teeth which sequentially squeeze the teeth into alignment. Simple cases can be treated in under a year.

2. Fixed appliances:

- Six Month Smiles ${ }^{\circledR}$ - this is a straight wire orthodontic system which consists of crystal clear labial brackets and tooth coloured super elastic NiTi wires that level and align the teeth that show in the smile but no attempt is made to correct any posterior molar malocclusions. The bracket positions are predetermined by a technician and are delivered using a carrier tray. Most cases can be completed
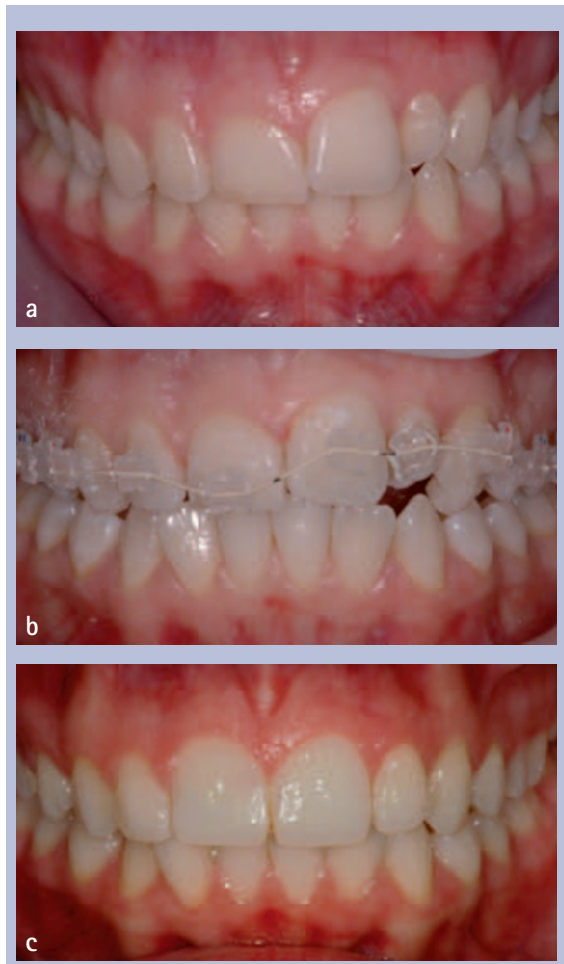

Fig. 1 Case study - short-term costmetic orthodontic patient (a) before treatment; (b) with Six Month Smiles ${ }^{\circledR}$ clear braces fitted; (c) post-treatment

in and around six months.

- Sectional lingual braces - the development of low profile brackets, like STB brackets, allows simple lingual orthodontics involving intercanine bracketing to level and align the anterior incisors. The brackets can also be delivered in prepositioned carrier trays. These treatments can be completed in a few months.

\section{RETENTION}

Following any adult orthodontic treatment, including comprehensive care, lifelong retention is imperative to avoid relapse. This can be achieved by either using a removable appliance such as a clear essix retainer or by placing a lingual bonded wire retainer. The movements undertaken with short-term orthodontics are predominately tipping movements rather than bucco lingual root torquing which takes much longer and falls outside the time parameters of short-term orthodontics. For this reason post-treatment retention becomes even more critical.

\section{Case study}

Christine, aged 23, is a typical short-term orthodontic patient who is seeking smile correction. She has declined traditional orthodontics as she was quoted over a year and was not prepared to have her teeth aggressively prepared. She was treated with Six Month Smiles ${ }^{\circledR}$ clear braces and composite incisal edge bonding in around five months.

Anoop Maini will be speaking on this subject on Saturday 27 April at the 2013 British Dental Conference and Exhibition, held at ExCel London. 